\title{
Intraoperative Neurophysiological Monitoring : A Review of Techniques Used for Brain Tumor Surgery in Children
}

\author{
Keewon Kim, M.D., Ph.D., ${ }^{1}$ Charles Cho, M.D., ${ }^{2}$ Moon-suk Bang, M.D., Ph.D., ${ }^{1}$ Hyung-ik Shin, M.D., Ph.D., \\ Ji-Hoon Phi, M.D., Ph.D., ${ }^{2}$ Seung-Ki Kim, M.D., Ph.D. ${ }^{3}$ \\ Department of Rehabilitation Medicine,' Seoul National University Children's Hospital, Seoul National University College of Medicine, Seoul, \\ Korea \\ Department of Neurology, ${ }^{2}$ Stanford University, Stanford, CA, USA \\ Department of Neurosurgery, ${ }^{3}$ Seoul National University Children's Hospital, Seoul National University College of Medicine, Seoul, Korea
}

Intraoperative monitoring (IOM) utilizes electrophysiological techniques as a surrogate test and evaluation of nervous function while a patient is under general anesthesia. They are increasingly used for procedures, both surgical and endovascular, to avoid injury during an operation, examine neurological tissue to guide the surgery, or to test electrophysiological function to allow for more complete resection or corrections. The application of IOM during pediatric brain tumor resections encompasses a unique set of technical issues. First, obtaining stable and reliable responses in children of different ages requires detailed understanding of normal ageadjusted brain-spine development. Neurophysiology, anatomy, and anthropometry of children are different from those of adults. Second, monitoring of the brain may include risk to eloquent functions and cranial nerve functions that are difficult with the usual neurophysiological techniques. Third, interpretation of signal change requires unique sets of normative values specific for children of that age. Fourth, tumor resection involves multiple considerations including defining tumor type, size, location, pathophysiology that might require maximal removal of lesion or minimal intervention. IOM techniques can be divided into monitoring and mapping. Mapping involves identification of specific neural structures to avoid or minimize injury. Monitoring is continuous acquisition of neural signals to determine the integrity of the full longitudinal path of the neural system of interest. Motor evoked potentials and somatosensory evoked potentials are representative methodologies for monitoring. Free-running electromyography is also used to monitor irritation or damage to the motor nerves in the lower motor neuron level : cranial nerves, roots, and peripheral nerves. For the surgery of infratentorial tumors, in addition to free-running electromyography of the bulbar muscles, brainstem auditory evoked potentials or corticobulbar motor evoked potentials could be combined to prevent injury of the cranial nerves or nucleus. IOM for cerebral tumors can adopt direct cortical stimulation or direct subcortical stimulation to map the corticospinal pathways in the vicinity of lesion. IOM is a diagnostic as well as interventional tool for neurosurgery. To prove clinical evidence of it is not simple. Randomized controlled prospective studies may not be possible due to ethical reasons. However, prospective longitudinal studies confirming prognostic value of IOM are available. Furthermore, oncological outcome has also been shown to be superior in some brain tumors, with IOM. New methodologies of IOM are being developed and clinically applied. This review establishes a composite view of techniques used today, noting differences between adult and pediatric monitoring.

Key Words : Monitoring, Intraoperative · Brain neoplasm · Neurosurgery · Child.

- Received : April 5, 2018 -Accepted : April 16, 2018

- Address for reprints : Seung-Ki Kim, M.D., Ph.D.

Department of Neurosurgery, Seoul National University Children's Hospital, Seoul National University College of Medicine, 101 Daehak-ro, Jongno-gu, Seoul 03080, Korea Tel : +82-2-2072-3084, Fax : +82-2-744-8459, E-mail : nsthomas@snu.ac.kr

This is an Open Access article distributed under the terms of the Creative Commons Attribution Non-Commercial License (http://creativecommons.org/licenses/by-nc/4.0) which permits unrestricted non-commercial use, distribution, and reproduction in any medium, provided the original work is properly cited. 


\section{INTRODUCTION}

Intraoperative neurophysiological monitoring (IOM) for neurosurgery is rapidly expanding; and, gaining increasing interest by both clinicians and researchers. Techniques, software, and hardware also have advanced over decades. However, there is disproportionately less research in pediatric brain tumor surgery compared to vascular, endovascular, spine, peripheral nerve cases, as well as in the adult literature. Neurophysiology in young children differs from that of adults. Depending on the age, responses are not as readily elicited and signals are not as stable as in adults ${ }^{20,26)}$.

In addition, monitoring certain complex brain functions is not as straightforward as measuring sensory and motor pathways which are well established to monitor the spinal cord ${ }^{12)}$. Eloquent functions, such as vision, hearing, speech, cranial nerve function, are more complex and require specialized methods and equipment. Also, monitoring of parenchymal tumor resection requires some expert judgement. For example, during tumor resection, for curative purposes, removal should be maximized whereas for functional consideration, removal should be minimized $^{54)}$. Numerous factors are considered coinciding with IOM : histopathology, age, function, lesion location, or others.

In this article, IOM for brain tumor resection will be described from various perspectives. Brief history of IOM, clinical evidence, basic principles and techniques, and specific considerations for tumors and pediatrics will be discussed.

\section{HISTORY OF INTRAOPERATIVE NEUROPHYSIOLOGICAL MONITORING}

Although several other departments (orthopaedics, otolaryngology, cardiac surgery, interventional neuroradiology, plastic and reconstructive surgery) may use IOM to prevent neurological injury and guide their surgery, main history of IOM is a part of neurosurgery history. Penfield, also having been referred to "the greatest living Canadian", employed direct cortical stimulation during epilepsy surgery as a surrogate test of cortical function $^{30,56)}$. His attempt was the beginning of IOM and provided the first insight to monitor human brain function in an anesthetized subject. Thereafter, electrocorticography, evoked potentials, electromyography, and nerve conduction studies were applied to identify neurophysiological function and epileptogenic regions during surgery ${ }^{30,38)}$. Those techniques are being applied ever since for epilepsy surgery although details have been modified recently, which will be dealt in techniques for direct cortical stimulation below.

Subsequently, intraoperative electroencephalography (EEG) was used to monitor cerebral ischemia during cardiac surgery $^{64,75,77)}$, which is still a good indicator in cerebrovascular surgery. Also, sensory and motor evoked potentials have been applied in spinal cord monitoring. Monitoring for the cord accumulated class 1 evidence, specifically for scoliosis corrections, and has become the standard of care in practice earlier than other surgeries. The reason is that these methods demonstrate 1) uniform electrophysiological changes across different types of spine surgeries, and 2) neurological sequelae from cord injury is grievous in functional aspects. Somatosensory evoked cc since $1970 \mathrm{~s}^{44-46,49)}$. In 1980s, motor evoked potentials (MEPs) were employed to evaluate the corticospinal tract (CST). Initially, transcranial magnetic stimulation was applied to the cortex, which is being used outside operation rooms at now. However, it was not feasible in operation rooms or for patients under general anesthesia. Later, Burke developed transcranial electrical stimulation methods which is being used today under general anesthesia ${ }^{4,27)}$. SEPs and MEPs are basic and integral parts of IOM of various surgeries so far.

Brainstem auditory evoked potentials (BAEPs) and electromyography (EMG) was integrated into IOM to monitor the auditory pathway from the periphery to auditory cortex and the cranial nerves, especially for infratentorial lesions ${ }^{41)}$. Other specific techniques such as root monitoring using pedicle screw stimulations have been introduced later ${ }^{36)}$. Finally, neurosurgery could cover the cortex, brainstem, spinal cord, roots and peripheral nerves to be monitored intraoperatively.

The development of IOM was facilitated by advances in commercial multi-channel IOM equipment. We cannot deny that the growth of hardware companies has promoted the development of IOM techniques and its clinical dissemination. Prior to 1981 when commercial IOM equipment became available, neurophysiologists had to devise their own equipment or modify EEG or EMG machines for intraoperative use.

Anesthesia also plays a key role in IOM. General anesthesia seeks to achieve analgesia, amnesia, immobility, hypnosis, and paralysis, all of which suppress neural activity. Neurosurgery under heavy sedation and deep general anesthesia can suppress meaningful neural activities and preclude IOM. Advances in 
total intravenous anesthesia (TIVA) have improved monitoring consistency. EEG changes with the level of anesthesia in a characteristic sequence, and with a distinct manner for each agent ${ }^{58)}$. Occasionally, SEPs are significantly suppressed by halothane inhalation anesthesia; and, can show decreased amplitude and increased latency in parallel with depressed of EEG ${ }^{2}$; BAEPs include multiple waves that reflect multiple synapses in the pathway and are progressively influenced by anesthesia ${ }^{14,29)}$; MEP is usually more resistant to anesthesia changes but more susceptible to neuromuscular blockade because it involves neuromuscular junction. Muscle relaxant can completely abolishes MEP and halothane inhalation agents at higher levels also may exert non-linearly dose-dependent suppression on $\mathrm{MEP}^{33,68)}$. TIVA, which usually combines propofol and opioids, demonstrates the most consistent and least obstructive sedation for IOM monitoring ${ }^{5,32,35,55,74)}$. Application of TIVA enabled neurophysiologists to obtain reliable responses.

\section{Clinical evidence and considerations}

Prognostic value of IOM was demonstrated in a systematic review $^{52)}$. Sensitivity and specificity can be increased by combining multiple modalities ${ }^{24,37)}$. In some specific conditions, IOM showed utility in preventing neurologic deficit, as an interventional tool $^{31)}$. Admittedly, further evidence or knowledge as for clinical utility of IOM should be explored. However, current level of evidence, expert opinion, and consensus indicate that IOM is beneficial and non-investigational for brain, brainstem, spinal cord, and cerebrovascular surgeries ${ }^{66)}$.

A special consideration for brain tumor is that there is an unavoidable compromise between maximal removal of tumor versus minimal removal of nerves. With certain tumor types and grades where total or near-total resection changes outcomes, the use of IOM to guide aggressive resection while minimizing new neurological deficits is important. Several studies demonstrated functional sequelae from brain tumor surgery were less with IOM than that without $\mathrm{IOM}^{3,15)}$. Concern about oncological outcome may remain with application of IOM. Of note, two previous studies revealed that in resection of low grade glioma, surgery using IOM could achieve higher rate of subtotal removal with $\mathrm{IOM}^{7,15)}$.

\section{PRINCIPLES AND TECHNIQUES}

\section{Monitoring and mapping}

IOM is defined as 'electrophysiological methodology to evaluate functional status of the nervous system during surgery and its nomenclature (intraoperative "monitoring") alludes as if it is solely for monitoring. However, IOM comprise two distinct functions : monitoring and mapping. Monitoring indicates continuous acquisition of neural signals to assess the integrity of the nervous system. Mapping identifies neural structures within the field of operation to avoid or minimize neural damage. Most of the electrophysiological tools can be employed. This classification applies to any neural parts (brain, cord, or nerve root) or clinical conditions (tumor, epilepsy, or anomaly). Also, this classification does not depend on specific electrophysiology employed; for example, SEP can be employed to "monitor" the sensory nervous system during scoliosis surgery whereas SEP can also be employed to "map" dorsal column of the spinal cord during intramedullary tumor surgery.

\section{MEP}

MEP directly activate and monitor motor pathways. There are different methodologies but the most common technique is by stimulating CST and recording responses at the spinal cord or muscles. Because MEP is generated and conveyed via the pyramidal tract, other motor systems such as basal ganglia or cerebellum are not evaluated from it. Stimulation is usually triggered by transcranial electrical stimulation via surface or subdermal needle electrodes on the scalp ${ }^{40)}$. It elicits excitation of corticospinal projections at various levels; just beneath the motor cortex, internal capsule, or pyramidal decussation" (Fig. 1). In order to isolate the side of interest, the stimulation parameters can be adjusted to avoid deeper structures. In cases where evaluation of the motor cortex is critical, direct cortical MEP (dcMEP) stimulation can be applied directly to the cortex using strip or grid electrode placed on the cortex can be considered. Using transcranial MEP, the montage of electrodes and intensity of current or voltage determines the depth where signals are generated; if stimulation is intense enough or electrodes are arranged at wide interval bilaterally, evoked potentials arise at the level of foramen magnum and propagate downward, thereby skipping the corticospinal tracts within the cerebrum. Appropriate intensity of stimulation current and appropriate arrangement of electrode should be determined for each individual case. 


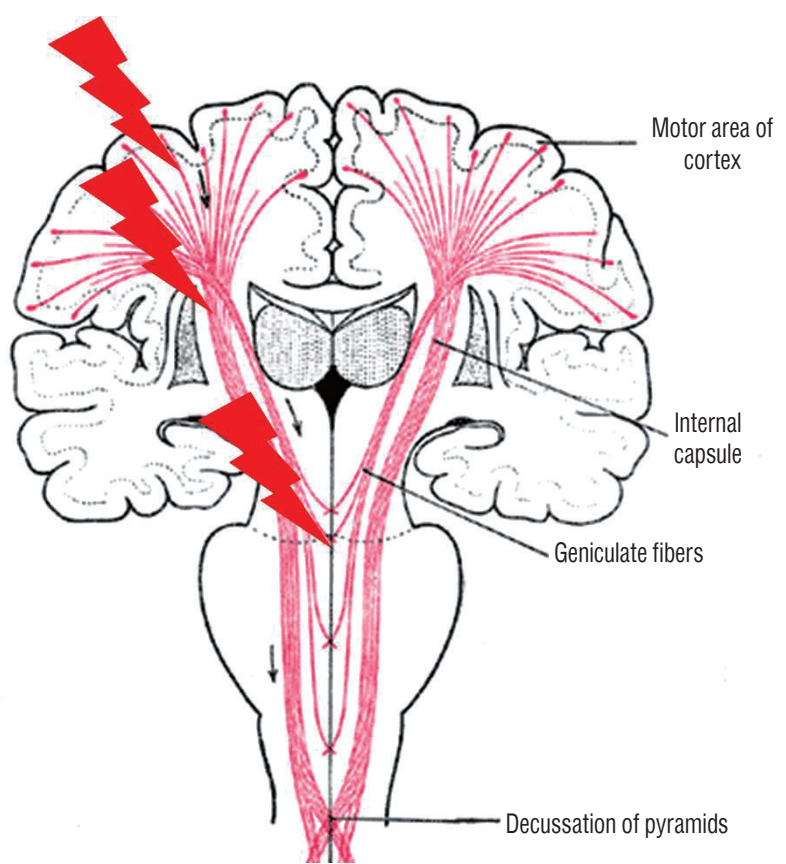

Fig. 1. Generation of motor evoked potentials at different levels of brain. Depending on the intensity of stimulation and the montage of electrode, motor evoked potentials are generated at different levels of brain. Superficial white mater just beneath the motor cortex, deep white matter of internal capsule, and pyramidal decussation are known to be major sites to be excited.

However, effective and stable response is not readily achieved even with strong currents only, especially in pediatric patients. Additional stimulation techniques can facilitate MEPs to optimize MEP monitoring. Two techniques are temporal facilitation and spatial facilitation. Temporal facilitation applies multipulse train stimulations usually composed of 4 to 5 pulses ${ }^{10)}$. Spatial facilitation applies peripheral tetanic stimulation prior to $\mathrm{MEP}^{20)}$. Combination of spatial and temporal facilitation can induce more reliable $\mathrm{MEPs}^{20)}$. In our experience, however, optimized temporal stimulation alone was as effective as the combination technique and was more readily applicable without additional equipment.

In pediatric cases, obtaining stable MEP waveforms is more challenging. Depending on their age, their nervous system is not fully developed in terms of myelination or synaptogenesis. Partly due to the myelination status, the electrophysiological responses are more sensitive to anesthesia. And, due to body size, placing stimulators, recording electrodes, grounds can be limited and in some instances trigger large stimulation artifacts that obscure the signal of interest ${ }^{21)}$. Therefore, MEP in pediatric patients frequently requires more pulses and different profile of trains (inter-pulse interval, pulse width, or others).
A MEP can be recorded at the spinal cord level (D-wave or Iwaves) or muscles of interest (myogenic MEP). Myogenic MEP is much larger in amplitude because it travels through neuromuscular junction and thereby amplified. Myogenic MEP responses are partly non-linear and can be interpreted qualitatively, in many circumstances per rule of "all or none"72). Additional studies suggest complexity and number of turns can be quantified and tracked linearly. D-waves can be interpreted quantitatively ${ }^{42}$.

\section{SEP}

SEP is the most commonly applied technique in IOM. Practically, sensory tracts are proximate to motor and are used as a surrogate to prevent motor deficits. SEP monitoring is usually performed by stimulating the tibial nerve at the ankle or median nerve at the wrist and recording the potentials at the scalp overlaying the sensory cortex. Triggered proprioceptive sensory signals are fastest and most potent thus SEPs mainly reflect the integrity of the posterior column in the spinal cord ${ }^{25}$. Therefore, ulnar nerve can substitute the median to examine the upper limbs and cervical spine or brain; but, the sural nerve cannot replace the tibial nerve because it does not convey proprioceptive sense; sensory modalities other than proprioception such as touch, pain, and temperature are not readily assessed by SEP. Similarly to MEPs, epidural electrodes at the spinal cord as well as scalp electrodes can record SEP( ${ }^{1}$. Epidural or subdural SEPs or D-waves place electrodes directly on the posterior column. SEPs are semi-quantitative measures and thus its interpretation for warning criteria is more specific than that of MEP. In general, a $50 \%$ decrease in amplitude or $10 \%$ delay in latency is regarded as a critical change $e^{50,51)}$.

\section{Median SEP phase reversal and dorsal column mapping}

Phase reversal is a commonly used procedure to determine the physiological location of the central sulcus ${ }^{22,23)}$. The method is illustrated below (Fig. 2). A strip electrode is placed perpendicularly across the approximate sulcus. The median nerve is then stimulated to generate a near-field response at the sensory cortex. EPs recorded at each electrode of the strip electrode will demonstrate different waveforms according to its relative location to a SEP dipole in the post-central gyrus. As the directionality of the dipole changes across the motor cortex (from the sensory stimulation), the phase of waveforms reverses creating 

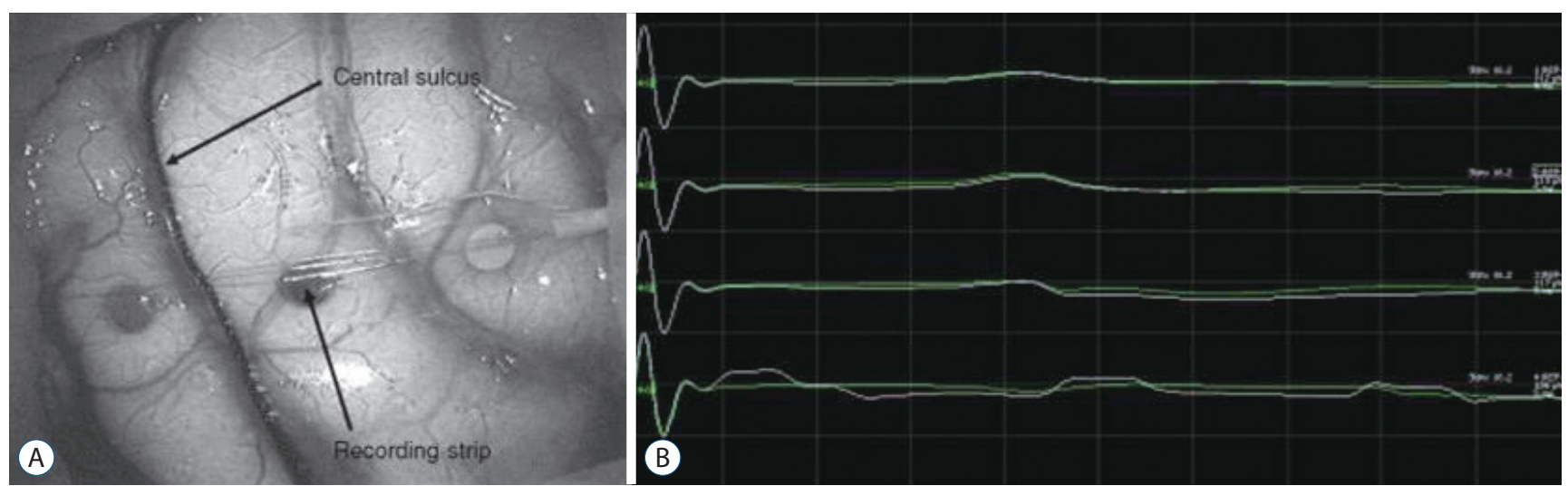

Fig. 2. Median sensory evoked potential (SEP) phase reversal. A : A strip electrode is placed perpendicularly across the approximate central sulcus. B : After stimulation of the contralateral median nerve at the wrist, median SEPs are recorded on the strip electrode; between the third and fourth electrode, SEP phase is reversed, which indicate physiological central sulcus.

the flipped waveform ${ }^{76}$.

Dorsal column mapping also utilizes SEP responses to determine the dorsal median raphe of the spinal cord. Accurate identification of the dorsal median raphe is important during myelotomy to preserve proprioception of each side, especially for intramedullary tumors where anatomical midline may not correspond with the physiologic midline due to distortion by tumor. There are three different approaches to localize the dorsal median raphe in IOM; stimulation of spinal cord at a fine intervals and recording retrograde sensory conduction at bilateral peripheral nerves (personal communication); stimulation of the peripheral nerves and recording orthograde sensory conduction on the spinal cord with strip electrode ${ }^{78}$; stimulation of spinal cord at fine intervals and recording SEPs on scalp to observe phase reversal ${ }^{65}$. In our experience, the third approach is more readily applicable because it does not need customized strip electrode and yields reliable responses within an acceptable duration.

\section{Free-running EMG}

EMG is regarded as a standard test for neuropathy and myopathy in clinic. Free-running EMG is the standard technique to monitor peripheral nerves, roots, or cranial motor nerves during surgery. Intraoperative EMG signals are activated immediately after cranial motor nerves are damaged or irritated; in contrast, abnormal EMG signals at outpatient clinic develop days to weeks after nerve injury. The mechanism of intraoperative EMG is different from conventional EMG and it still remains unclear. The duration, morphology, and persistence of EMG reflects severity of neural injury; the longer EMG train persists, the more likely neural deficits follow after surgery ${ }^{57)}$; the high frequency of sinusoidal, symmetric sequence of EMG discharges implies probable neural injury ${ }^{61}$. However, injuries from sharp transection or gradual ischemia may not revoke any EMG signal.

\section{Direct cortical stimulation (DCS) and direct subcortical stimulation (DSCS)}

Neuro-navigation system provides 3-D image position guidance of anatomical location (Fig. 3). However, this navigation system is based on images taken prior to operation and cannot reflect anatomical shifts during operation from positioning, tissue removal, or edema. Also, it does not provide neurophysiological or functional information. Neuro-navigation alone cannot differentiate whether a region being targeted contains functional neural substrate or not. DCS or DSCS provides complementary information allowing surgeons to discern whether surgical field of interest involves functional motor cortex or CST or how far it is from CST to be preserved in a quantitative way (Fig. 4). However, motor responses are not easily obtained from the cortex or subcortex by single pulse of stimulation. Historically, the Penfield technique has been a standard for DCS which employs tetanic $(50-60 \mathrm{~Hz})$ of single-pulse stimulation. However, multi-pulse short-train stimulation was introduced as more efficacious technique for DCS ${ }^{73)}$ with less risk of seizure ${ }^{70)}$ (Fig. 5). DSCS with multi-pulse stimulation also requires lower threshold intensity than the Penfield technique ${ }^{71)}$. In children, determination of stimulation paradigm is more important because of difficulty to elicit motor responses, directly relevant to the immaturity of the nervous system. Multi-pulse train stim- 


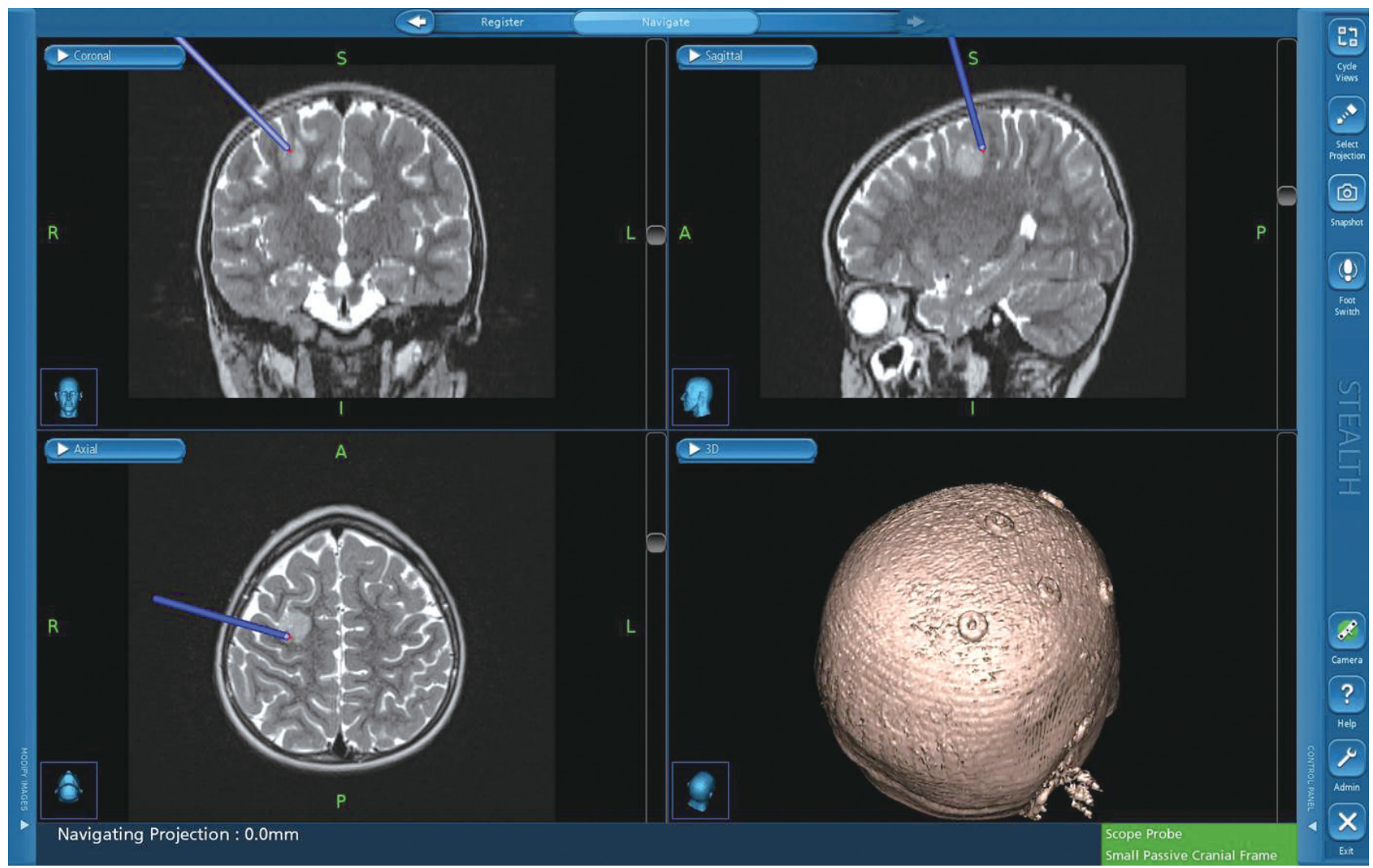

Fig. 3. Neuro-navigation for brain tumor surgery. Neuro-navigation system provides 3-D image position guidance of anatomical location. However, it cannot reflect anatomical shifts during operation from positioning, tissue removal, or edema and it does not provide neurophysiological or functional information.

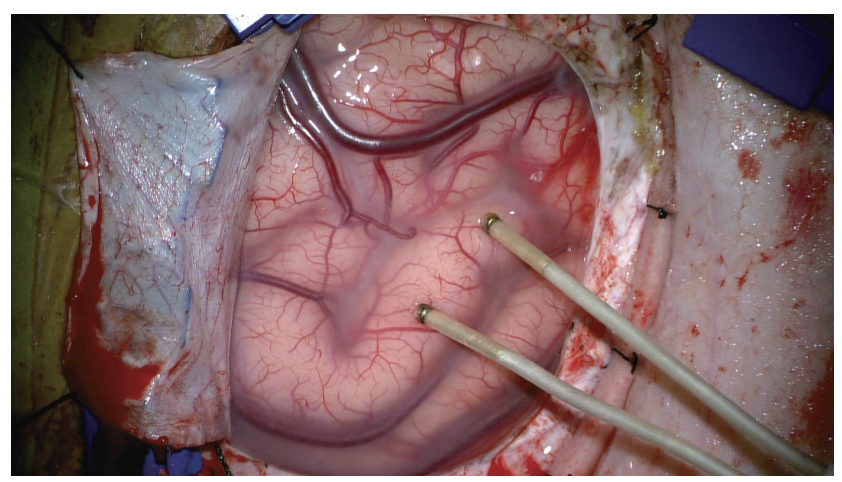

Fig. 4. A case of direct cortical stimulation. To map the motor cortex in proximity to a lesion, direct cortical stimulation is applied.

ulation, modified from adult protocol, should be delivered.

DSCS threshold linearly correlates with distance between stimulation site and the $\mathrm{CST}^{53}$. One mm further from the CST approximately correlates with $1 \mathrm{~mA}$ incremental stimulation threshold. A recent technique that combines suction tip with stimulation probe, also known as "suction probe", is more eas- ily applicable $e^{59,63)}$.

\section{BAEP and other cranial nerve evaluation}

Monitoring or mapping of cranial nerves (CNs) or nuclei is an integral part of infratentorial brain tumor surgery. BAEP is performed by delivering acoustic stimuli of a clicking sound, which encompasses a wide range of frequencies, through earplugs and recording near- and far-field potentials from electrodes at the mastoid process or earlobes ${ }^{60)}$. Because BAEP is composed of multiple waves (near-field potentials, wave I and II, and farfield potentials, wave III to V, VI) of both sides, it is imperative to interpret the change of BAEP waves with neuro-anatomical consideration. Monitoring BAEP change are mainly focused on the 'amplitude reduction' of wave III and V, as well as the interwave latencies ${ }^{39)}$. However, attention to wave II or III may provide opportunity for earlier intervention to reversible damage.

Cranial motor nerves (oculomotor nerve to the inferior or medial rectus [CN III], trochlear nerve to the superior oblique [CN IV], trigeminal nerve to the masseter [CN V], abducens 
nerve to the lateral rectus [CN VI], facial nerve [CN VII], glossopharyngeal nerve to stylopharyngeus muscle [CN IX], vagus nerve to laryngeal muscles [CN X], accessory nerve to the trapezius [CN XI], and hypoglossal nerve to the genioglossus $[\mathrm{CN}$ $\mathrm{XII}]$ ) can be monitored by free-running EMG as well as transcranial corticobulbar MEP. Vagus nerve can be evaluated by recording MEP of the laryngeal muscles via surface electrode attached on endotracheal tube and hypoglossal nerve can be via paired needle electrodes inserted in the tongue muscle. Freerunning EMG for bulbar muscles is performed as described above, which is identical to that for limb muscles using cathode and anode electrodes. However, corticobulbar MEP should be conducted with extra caution compared with corticospinal MEP because bulbar muscles are in proximity to stimulation electrodes on the scalp. To rule out direct spread of current, a pair of stimulations are applied at short intervals, composed of single-pulse stimulation and multi-pulse stimulation respectively. Absence of response from single-pulse stimulation and presence of response from multi-pulse stimulation indicate that the response transmits via the corticobulbar tract rather than direct current spread $^{13)}$. In addition, cranial nuclei, especially facial colliculus, can be also mapped in the brainstem during surgery by direct stimulation ${ }^{6,17,43,69)}$. Diligent application of IOM in brainstem surgery can reduce, or at least weigh in advance, the risk of serious functional deficits such as dysphasia, dysphonia, facial palsy, or hearing loss.

\section{CONSIDERATIONS BY TUMOR LOCATIONS}

In this section, the application of various modalities of IOM will be described per location of brain tumors, which is mainly based on literature, anatomical network connection principles, as well as the authors' experience.

\section{Supratentorial tumors}

Supratentorial tumors frequently involves eloquent areas : sensory, motor, visual, and language areas (Fig. 6). Awake sur-

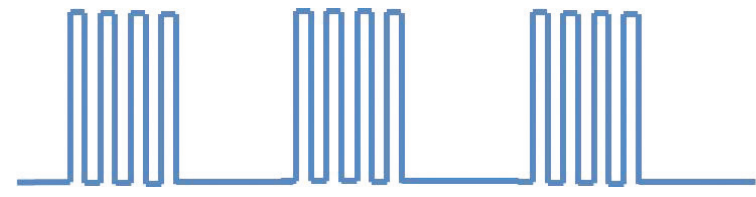

(A)
Multi-pulse train stimulation

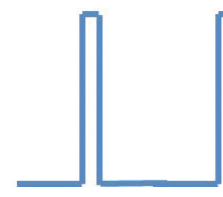

(B)

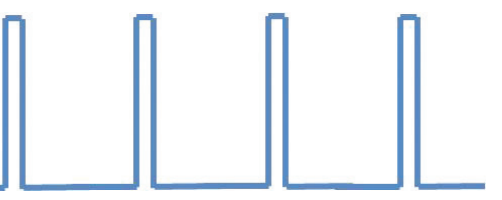

Single-pulse stimulation

Fig. 5. Stimulation techniques for motor evoked potentials. Schematic illustration shows multi-pulse train stimulation (A) and single-pulse stimulation (Penfield technique) (B). Multi-pulse train stimulation requires lower intensity of stimulation and poses less risk of seizure than single-pulse stimulation.
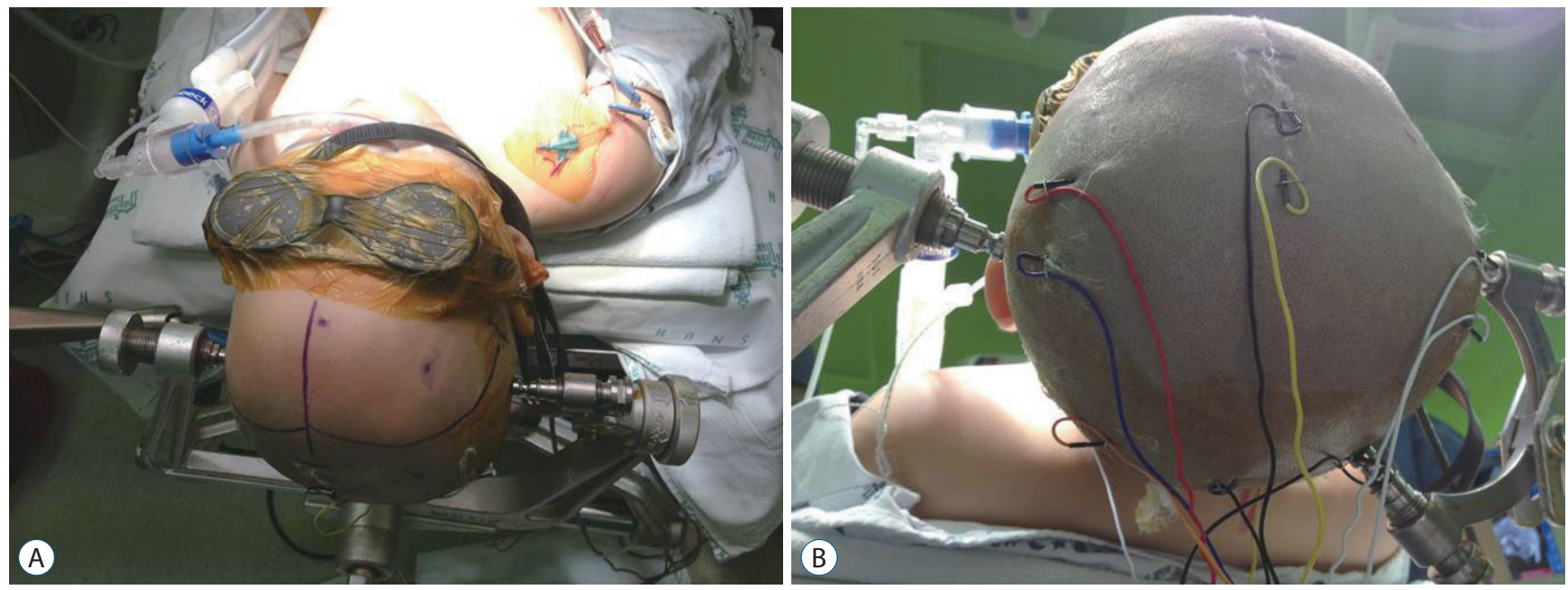

Fig. 6. Setting for visual evoked potential. Visual evoked potential can monitor the visual pathway. A : Light stimulation is applied via goggles. B : Needle electrodes are placed at the scalp of the occipital cortex and record visual evoked potentials. 
gery is not an option for young pediatric patients. Sensory and motor systems include "peripheral interface", to which the authors prefer to refer, to stimulate or record in the body : i.e., muscles and sensory nerve endings. However, other eloquent systems do not include input or output interfaces in the body, which makes fundamental difference to IOM.

First, sensory and motor cortex can be mapped by using median SEP phase reversal. Motor cortex can be further mapped by DCS to reveal which part corresponds to which motor function.

Then, the sensory and motor systems can be monitored by SEP and transcranial MEP. MEP stimulation intensity should be calibrated not to bypass the region of interest in the cerebrum; if stimulation intensity is strong enough, MEP generates at the deep white/grey matter or brainstem; if stimulation intensity is too weak, MEP is not elicited at a stable manner; only appropriate intensity of stimulation evokes MEP at the level of cortex. In cases when generation of MEP must be pursued at the motor cortex, dcMEP using strip electrode on the cortex may be an option. On the contrary, SEP comprises signals from the sensory cortex and can be monitored for cortical function as such.

Subcortical lesions can be greatly helped by DSCS using suction probes. As describe above, continuous monitoring of threshold intensity for SCDS informs distance to the SCT. Together with neuro-navigation system, SCDS can help to preclude inadvertent neural damage leading to functional deficits.

\section{Infratentorial tumors}

Infratentorial tumors such as medulloblastoma, brainstem glioma, or ependymoma have high incidence and morbidity in the pediatric population. These infratentorial tumors are very challenging from the perspective of IOM; routine SEP or MEP from four extremities alone cannot predict post-operative functional deficits involving cerebellum or cranial nerves. Monitoring of the cerebellum is still a quest for research. However, by combining various methodologies, the brainstem can be monitored or mapped. BAEP would be a standard tool for brainstem monitoring, with validated sensitivity and specificity ${ }^{47)}$. Freerunning EMG and corticobulbar MEP can be employed to monitor the cranial nerves at risk. Facial nerves can be monitored by both EMG and MEP of the facial muscles. Trigeminal nerve can be monitored from EMG and MEP of the masseter or temporalis. If lesions involve the medulla oblongata and swallowing function is at risk, laryngeal muscle MEP for monitoring of the vagus nerve should be considered. Lesions that place CN III, IV, and VI at risk may require EMG of the extra-ocular muscles. The accessory nerve or hypoglossal nerve can be also monitored at the trapezius or genioglossus by EMG and MEP.

Deploying multi-modal monitoring can cover substantial regions of brainstem (Fig. 7). Most IOM signals are more sensitive than specific; thus, in many cases, temporary or intermittent changes in a single modality may require additional analysis and corroboration. All the electrophysiological signals should be interpreted in the context of neuroanatomy and surgical procedure.

\section{Cerebellopontine angle tumor}

Cerebellopontine angle tumor surgical techniques have advanced in conjunction with IOM of the auditory pathways and facial nerves. BAEP with facial EMG/MEP is a mainstay of IOM. BAEP is a very sensitive tool for monitoring the auditory system including the cochlear nerve. Free-running EMG and corticobulbar MEP for facial muscles are also an effective tool for monitoring the facial nucleus and facial nerve. Although the vestibular nerve courses together with the cochlear nerve, electrophysiological differentiation between them is seldom required because anatomical localization suffices for clinical purposes; however, if cochlear nerve is isolated, IOM can directly record nerve action potentials from the nerve $e^{8,18,19,48)}$. Without additional installation to facial MEP monitoring, facial EMG can be observed; thus, facial EMG and MEP are almost always monitored together. Whenever the facial nerve is irritated, facial EMG occurs. Neurophysiologist should be prudent about its interpretation based on its waveform : duration, frequency, symmetry, and amplitude ${ }^{61)}$. To corroborate any possible damage to the facial nerve, the facial MEP can check the integrity of the facial motor system.

\section{DISCUSSION}

\section{Limitations and future studies}

This review covers several IOM modalities and some practical applications. These techniques developed over multiple decades and additional new methods and applications are still being introduced. The clinical volume of IOM cases is increasing exponentially. The authors expect new techniques and better neurophysiologic understanding will add to the utility of IOM 


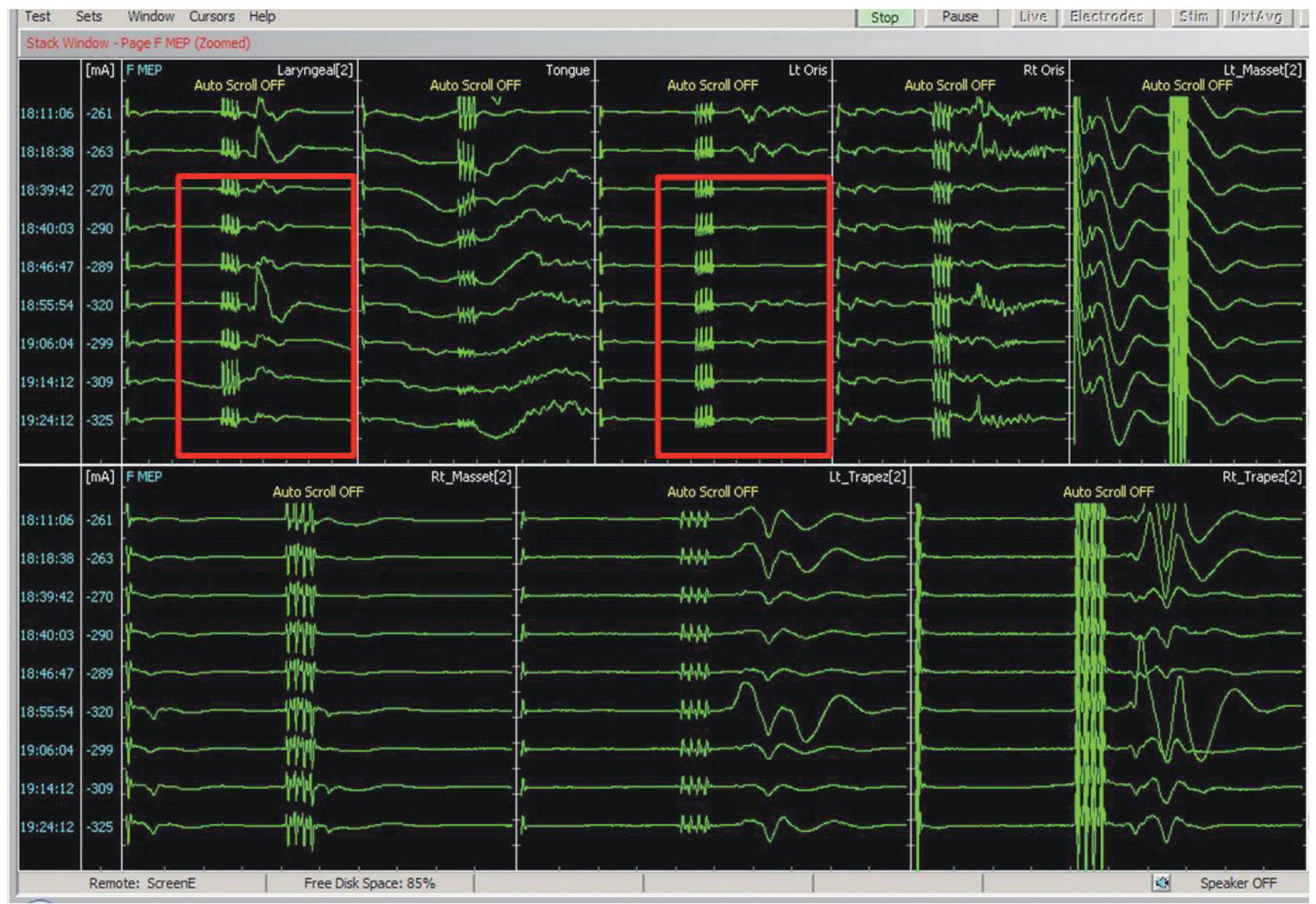

Fig. 7. Cranial nerve monitoring for infratentorial tumor surgery. For intraoperative monitoring of infratentorial tumor surgery, multi-modal monitoring should be combined including monitoring for the cranial nerves at risk. In the figure, motor evoked potentials (MEPs) of cranial nerve V, VII, X, XI, and XII are being recorded. Red boxes indicate disappearance of left laryngeal and left facial muscle MEPs. Of note, single pulse stimulation is immediately followed by multi-pulse stimulation, to rule out current spread in corticobulbar MEP.

in the future.

An area of weakness of current IOM modalities is that they depend on the peripheral interface. Eloquent neural systems that do not directly integrate peripheral motor or sensory system such as language areas or cerebellum cannot be monitored. Currently, the language area is localized by preoperative functional magnetic resonance image or mapped as part of an 'asleepawake-asleep' craniotomy. There are a few novel attempts to electrophysiologically monitor non-motor, non-sensory, eloquent functions. For example, cortical EP at Broca's area was elicited by stimulation at Wernicke's area ${ }^{62)}$. Vedran Deletis showed that direct or indirect stimulation of motor language cortex induced delayed response in laryngeal muscles ${ }^{11)}$. In children, cerebellar cognitive affective syndrome, also known as cerebellar mutism, is also of great clinical concern after surgery of infratentorial tumors. However, current modalities in IOM fails to moni- tor the cerebellum.

This review delineated that the pediatric population is qualitatively different from adults in terms of intraoperative neurophysiology. The nervous system is immature; myelination and synaptogenesis are still under development, especially under the age of 3 years ${ }^{16,34)}$. Anthropometry of young children is different; shock artifact is more problematic due to the close proximity of stimulation to recording electrodes. The fontanel is open until 18 months old, which requires that montage of electrode for stimulation and recording must be modified accordingly. The effect of anesthesia is also different; depression of evoked potential due to halothane anesthesia is more pronounced in children $^{67)}$. Therefore, we must develop stimulation and recording techniques different from routine adult paradigms and establish new tailored criteria to interpret IOM responses in children ${ }^{28)}$.

Finally, there are very few high levels of evidence for the use 
of IOM, and the various techniques beyond case series, retrospective studies, uncontrolled longitudinal observations. It is fundamentally because IOM is a diagnostic as well as an interventional tool; i.e., IOM provides data that must be used immediately to react to avoid new post-operative neurological deficits. When findings from IOM indicate that nerves being manipulated are at risk of injury, it is unethical to not to intervene, thereby creating type 2 errors of false negative outcome with any IOM change. Conducting randomized controlled trial, with IOM versus without IOM, cannot be conducted for these ethical reasons. Retrospective review comparing clinical outcomes of operations with IOM versus without IOM is weaker evidence because IOM is usually applied in more complicated surgeries which naturally have higher risks and poorer outcomes. Historical comparison with operations before introduction of IOM to current cases with IOM is also not valid because advances in imaging, anesthesia, surgical techniques, or others make these comparisons inaccurate.

\section{CONCLUSION}

IOM is an important neurophysiological tool to minimize sequelae during surgical procedures that put neurological tissue at risk. Historical and current data suggests IOM alters the risk profile of brain tumor surgery and that neuro-oncological outcome can be improved with monitoring. Pediatric cases can be also aided by IOM. However, it requires additional consideration because intraoperative neurophysiology is different in children. The underlying methodologies are similarly applied, but the stimulation paradigm or interpretation criteria should be age-adjusted.

\section{CONFLICTS OF INTEREST}

No potential conflict of interest relevant to this article was reported.

\section{INFORMED CONSENT}

This type of study does not require informed consent.

\section{- Acknowledgements}

This work (2017R1A2B2008422) was supported by Mid-career Researcher Program through NRF grant funded by the Korea government (Ministry of Science and ICT).

\section{References}

1. Anderson $\mathrm{S}$, Loughnan $\mathrm{B}$, Hetreed $\mathrm{M}$ : A technique for monitoring evoked potentials during scoliosis and brachial plexus surgery. Ann R Coll Surg Engl $72: 321-323,1990$

2. Angel A, GRATTON DA : The effect of anaesthetic agents on cerebral cortical responses in the rat. Br J Pharmacol 76 : 541-549, 1982

3. Brell M, Ibáñez J, Caral L, Ferrer $E$ : Factors influencing surgical complications of intra-axial brain tumours. Acta Neurochir (Wien) 142 : 739 750, 2000

4. Burke D, Hicks R, Stephen J, Woodforth I, Crawford M : Assessment of corticospinal and somatosensoty conduction simultaneously during scoliosis surgery. Electroencephalogr Clin Neurophysiol 85 : 388-396, 1992

5. Calancie B, Harris W, Broton JG, Alexeeva N, Green BA : "Threshold-level" multipulse transcranial electrical stimulation of motor cortex for intraoperative monitoring of spinal motor tracts: description of method and comparison to somatosensory evoked potential monitoring. J Neurosurg 88 : 457-470, 1998

6. Chang SD, López JR, Steinberg GK : Intraoperative electrical stimulation for identification of cranial nerve nuclei. Muscle Nerve 22 : 1538-1543, 1999

7. De Witt Hamer PC, Robles SG, Zwinderman AH, Duffau H, Berger MS : Impact of intraoperative stimulation brain mapping on glioma surgery outcome: a meta-analysis. J Clin Oncol 30 : 2559-2565, 2012

8. Debatisse D, Pralong E, Guerit J, Bisdorff A : Recording click-evoked myogenic potentials (CEMPs) with a setup for brainstem auditory evoked potentials (BAEPs). Neurophysiol Clin 35 : 109-117, 2005

9. Deletis $V$, Shils J : Neurophysiology in neurosurgery. New York : Elsevier, 2002, pp25-51

10. Deletis V, Rodi Z, Amassian VE : Neurophysiological mechanisms underlying motor evoked potentials in anesthetized humans. Part 2. relationship between epidurally and muscle recorded MEPs in man. Clin Neurophysiol $112: 445-452,2001$

11. Deletis V, Rogić M, Fernández-Conejero I, Gabarrós A, Jerončić A : Neurophysiologic markers in laryngeal muscles indicate functional anatomy of laryngeal primary motor cortex and premotor cortex in the caudal opercular part of inferior frontal gyrus. Clin Neurophysiol 125 : 19121922, 2014

12. Deletis $V$, Sala F : Intraoperative neurophysiological monitoring of the spinal cord during spinal cord and spine surgery: a review focus on the corticospinal tracts. Clin Neurophysiol 119 : 248-264, 2008

13. Dong CC, MacDonald DB, Akagami R, Westerberg B, AlKhani A, Kanaan I, et al. : Intraoperative facial motor evoked potential monitoring with 
transcranial electrical stimulation during skull base surgery. Clin Neurophysiol 116 : 588-596, 2005

14. Dubois MY, Sato S, Chassy J, Macnamara TE : Effects of enflurane on brainstem auditory evoked responses in humans. Anesth Analg 61 : 898-902, 1982

15. Duffau $H$, Lopes $M$, Gatignol $P$, Mandonnet $E$, Taillandier L, Leroy $M$, et al. : 745 contribution of intraoperative corticosubcortical stimulations in surgery of low-grade gliomas: a comparative study between two series without and with functional mapping. Neurosurgery $55:$ 467, 2004

16. Eggermont $\mathrm{J}$ : Physiology of the developing auditory system in Sandra E Trehub, Bruce Schneider (eds) : Auditory development in infancy. New York : Springer, 1985, pp21-45

17. Eisner W, Schmid UD, Reulen HJ, Oeckler R, Olteanu-Nerbe V, Gall C, et al. : The mapping and continuous monitoring of the intrinsic motor nuclei during brain stem surgery. Neurosurgery 37 : 255-265, 1995

18. Ferber-Viart C, Dubreuil C, Duclaux R : Vestibular evoked myogenic potentials in humans: a review. Acta Otolaryngol $119:$ : 6-15, 1999

19. Ferber-Viart C, Duclaux R, Colleaux B, Dubreuil C : Myogenic vestibularevoked potentials in normal subjects: a comparison between responses obtained from sternomastoid and trapezius muscles. Acta Otolaryngol 117 : 472-481, 1997

20. Frei FJ, Ryhult SE, Duitmann E, Hasler CC, Luetschg J, Erb TO : Intraoperative monitoring of motor-evoked potentials in children undergoing spinal surgery. Spine (Phila Pa 1976) 32 : 911-917, 2007

21. Fulkerson DH, Satyan KB, Wilder LM, Riviello JJ, Stayer SA, Whitehead $W E$, et al. : Intraoperative monitoring of motor evoked potentials in very young children. J Neurosurg Pediatr 7 : 331-337, 2011

22. Goldring $S:$ A method for surgical management of focal epilepsy, especially as it relates to children. J Neurosurg 49 : 344-356, 1978

23. Goldring S, Gregorie EM : Surgical management of epilepsy using epidural recordings to localize the seizure focus: review of 100 cases. J Neurosurg $60: 457-466,1984$

24. Gunnarsson T, Krassioukov AV, Sarjeant R, Fehlings MG : Real-time continuous intraoperative electromyographic and somatosensory evoked potential recordings in spinal surgery: correlation of clinical and electrophysiologic findings in a prospective, consecutive series of 213 cases. Spine (Phila Pa 1976) 29 : 677-684, 2004

25. Halonen JP, Jones SJ, Edgar MA, Ransford AO : Conduction properties of epidurally recorded spinal cord potentials following lower limb stimulation in man. Electroencephalogr Clin Neurophysiol 74 : 161-174, 1989

26. Helmers SL, Hall JE : Intraoperative somatosensory evoked potential monitoring in pediatrics. J Pediatr Orthop 14 : 592-598, 1994

27. Hicks RG, Burke DJ, Stephen JP : Monitoring spinal cord function during scoliosis surgery with Cotrel-Dubousset instrumentation. Med J Aust $154: 82-86,1991$

28. Hwang H, Wang KC, Bang MS, Shin HI, Kim SK, Phi JH, et al. : Optimal stimulation parameters for intraoperative bulbocavernosus reflex in infants. J Neurosurg Pediatr 20 : 464-470, 2017

29. James MFM, Thornton C, Jones JG : Halothane anaesthesia changes the early components of the auditory evoked response in man. Br J An- aesth $54: 787,1982$

30. Jasper $\mathrm{H}$ : Electrocorticograms in man. Electroencephalogr Clin Neurophysiol $2: 16-29,1949$

31. Jimenez JC, Sani S, Braverman B, Deutsch H, Ratliff JK : Palsies of the fifth cervical nerve root after cervical decompression: prevention using continuous intraoperative electromyography monitoring. J Neurosurg Spine $3:$ : 92-97, 2005

32. Jones S, Harrison R, Koh K, Mendoza N, Crockard H : Motor evoked potential monitoring during spinal surgery: responses of distal limb muscles to transcranial cortical stimulation with pulse trains. Electroencephalogr Clin Neurophysiol 100 : 375-383, 1996

33. Kalkman CJ, Drummond JC, Ribberink AA : Low concentrations of isoflurane abolish motor evoked responses to transcranial electrical stimulation during nitrous oxide/opioid anesthesia in humans. Anesth Analg $73:$ 410-415, 1991

34. Levy SR : Somatosensory Evoked Potentials in Chiappa KH (ed) : Evoked potentials in clinical medicine, ed 3. Philadelphia : Lippincott-Raven, 1997, pp453-466

35. Lo YL, Dan YF, Tan YE, Nurjannah S, Tan SB, Tan CT, et al. : Intraoperative motor-evoked potential monitoring in scoliosis surgery: comparison of desflurane/nitrous oxide with propofol total intravenous anesthetic regimens. J Neurosurg Anesthesiol 18 : 211-214, 2006

36. Maguire J, Wallace S, Madiga R, Leppanen R, Draper V : Evaluation of intrapedicular screw position using intraoperative evoked electromyography. Spine (Phila Pa 1976) 20 : 1068-1074, 1995

37. Manninen PH, Patterson S, Lam AM, Gelb AW, Nantau WE : Evoked potential monitoring during posterior fossa aneurysm surgery: a comparison of two modalities. Can J Anaesth 41 : 92-97, 1994

38. Marshall C, Walker AE : Electrocorticography. Bull Johns Hopkins Hosp 85 : 344-359, 1949

39. Matthies C, Samii M : Management of vestibular schwannomas (acoustic neuromas): the value of neurophysiology for intraoperative monitoring of auditory function in 200 cases. Neurosurgery 40 : 459-466; discussion 466-468, 1997

40. Merton $\mathrm{P}$, Morton $\mathrm{H}$ : Stimulation of the cerebral cortex in the intact human subject. Nature $285: 227,1980$

41. Møller MB, Møller AR : Loss of auditory function in microvascular decompression for hemifacial spasm: results in 143 consecutive cases. J Neurosurg $63: 17-20,1985$

42. Morota N, Deletis V, Constantini S, Kofler M, Cohen H, Epstein FJ : The role of motor evoked potentials during surgery for intramedullary spinal cord tumors. Neurosurgery 41 : 1327-1336, 1997

43. Morota N, Deletis V, Epstein FJ, Kofler M, Abbott R, Lee M, et al. : Brain stem mapping: neurophysiological localization of motor nuclei on the floor of the fourth ventricle. Neurosurgery 37 : 922-929; discussion 929-930, 1995

44. Nash CL Jr, Brodkey J : Clinical application of spinal-cord monitoring for operative treatment of spinal disease. Cleveland: Case Western Reserve University, 1977, p140

45. Nash CL Jr, Lorig RA, Schatzinger LA, Brown RH : Spinal cord monitoring during operative treatment of the spine. Clin Orthop Relat Res (126) : 
100-105, 1977

46. Nash $\mathrm{CL} J$ r, Schatzinger $\mathrm{L}$, Lorig $\mathrm{R}$ : Intraoperative monitoring of spinal cord function during scoliosis spine surgery. J Bone Joint Surg Am 56 : 765, 1974

47. Neu M, Strauss C, Romstöck J, Bischoff B, Fahlbusch R : The prognostic value of intraoperative BAEP patterns in acoustic neurinoma surgery. Clin Neurophysiol 110 : 1935-1941, 1999

48. Nguyen $B H$, Javel $E$, Levine SC : Physiologic identification of eighth nerve subdivisions: direct recordings with bipolar and monopolar electrodes. Am J Otol 20 : 522-534, 1999

49. Nuwer $M$, Dawson $E$ : Intraoperative evoked potential monitoring of the spinal cord: enhanced stability of cortical recordings. Electroencephalogr Clin Neurophysiol 59 : 318-327, 1984

50. Nuwer MR, Aminoff M, Desmedt J, Eisen AA, Goodin D, Matsuoka S, et al. : IFCN recommended standards for short latency somatosensory evoked potentials. Report of an IFCN committee. International federation of clinical neurophysiology. Electroencephalogr Clin Neurophysiol $91: 6-11,1994$

51. Nuwer MR, Dawson EG, Carlson LG, Kanim LE, Sherman JE : Somatosensory evoked potential spinal cord monitoring reduces neurologic deficits after scoliosis surgery: results of a large multicenter survey. Electroencephalogr Clin Neurophysiol 96 : 6-11, 1995

52. Nuwer MR, Emerson RG, Galloway G, Legatt AD, Lopez J, Minahan R, et al. : Evidence-based guideline update: intraoperative spinal monitoring with somatosensory and transcranial electrical motor evoked potentials report of the therapeutics and technology assessment subcommittee of the American Academy of Neurology and the American Clinical Neurophysiology Society. Neurology 78 : 585-589, 2012

53. Ohue S, Kohno S, Inoue A, Yamashita D, Harada H, Kumon Y, et al. : Accuracy of diffusion tensor magnetic resonance imaging-based tractography for surgery of gliomas near the pyramidal tract: a significant correlation between subcortical electrical stimulation and postoperative tractography. Neurosurgery 70 : 283-293; discussion 294, 2012

54. Sawaya R, Hammoud M, Schoppa D, Hess KR, Wu SZ, Shi WM, et al. : Neurosurgical outcomes in a modern series of 400 craniotomies for treatment of parenchymal tumors. Neurosurgery 42 : 1044-1055; discussion 1055-1056, 1998

55. Pechstein U, Cedzich C, Nadstawek J, Schramm J : Transcranial highfrequency repetitive electrical stimulation for recording myogenic motor evoked potentials with the patient under general anesthesia. Neurosurgery 39 : 335-343; discussion 343-344, 1996

56. Penfield W, Boldrey E : Somatic motor and sensory representation in the cerebral cortex of man as studied by electrical stimulation. Brain 60 : 389-443, 1937

57. Prell J, Rampp S, Romstöck J, Fahlbusch R, Strauss C : Train time as a quantitative electromyographic parameter for facial nerve function in patients undergoing surgery for vestibular schwannoma. J Neurosurg 106 : 826832, 2007

58. Purdon PL, Sampson A, Pavone KJ, Brown EN : Clinical electroencephalography for anesthesiologists: part l: background and basic signatures. Anesthesiology 123 : 937-960, 2015
59. Raabe A, Beck J, Schucht P, Seidel K: Continuous dynamic mapping of the corticospinal tract during surgery of motor eloquent brain tumors: evaluation of a new method. J Neurosurg 120 : 1015-1024, 2014

60. Radtke RA, Erwin CW, Wilkins RH : Intraoperative brainstem auditory evoked potentials: significant decrease in postoperative morbidity. Neurology 39(2 Pt 1) : 187-191, 1989

61. Romstöck J, Strauss C, Fahlbusch R : Continuous electromyography monitoring of motor cranial nerves during cerebellopontine angle surgery. J Neurosurg 93 : 586-593, 2000

62. Saito T, Tamura M, Muragaki Y, Maruyama T, Kubota Y, Fukuchi S, et al. : Intraoperative cortico-cortical evoked potentials for the evaluation of language function during brain tumor resection: initial experience with 13 cases. J Neurosurg $121: 827-838,2014$

63. Schucht P, Seidel K, Beck J, Murek M, Jilch A, Wiest R, et al. : Intraoperative monopolar mapping during 5-ALA-guided resections of glioblastomas adjacent to motor eloquent areas: evaluation of resection rates and neurological outcome. Neurosurg focus 37 : E16, 2014

64. Sharbrough FW, Messick JM Jr, Sundt TM Jr : Correlation of continuous electroencephalograms with cerebral blood flow measurements during carotid endarterectomy. Stroke 4 : 674-683, 1973

65. Simon MV, Chiappa KH, Borges LF : Phase reversal of somatosensory evoked potentials triggered by gracilis tract stimulation: case report of a new technique for neurophysiologic dorsal column mapping. Neurosurgery 70 : E783-E788, 2012

66. Slimp JC : Electrophysiologic intraoperative monitoring for spine procedures. Phys Med Rehabil Clin N Am 15 : 85-105, 2004

67. Sloan $\mathrm{T}$ : Anesthesia and intraoperative neurophysiological monitoring in children. Childs Nerv Syst 26 : 227-235, 2010

68. Stone JL, Ghaly RF, Levy WJ, Kartha R, Krinsky L, Roccaforte P : A comparative analysis of enflurane anesthesia on primate motor and somatosensory evoked potentials. Electroencephalogr Clin Neurophysiol 84 : 180-187, 1992

69. Strauss C, Romstöck J, Nimsky C, Fahlbusch R : Intraoperative identification of motor areas of the rhomboid fossa using direct stimulation. J Neurosurg 79 : 393-399, 1993

70. Szelényi $A$, Joksimovic $B$, Seifert $V$ : Intraoperative risk of seizures associated with transient direct cortical stimulation in patients with symptomatic epilepsy. J Clin Neurophysiol 24 : 39-43, 2007

71. Szelényi A, Senft C, Jardan M, Forster M, Franz K, Seifert $V$, et al. : Intraoperative subcortical electrical stimulation : a comparison of two methods. Clin Neurophysiol 122 : 1470-1475, 2011

72. Tanaka Y, Kawaguchi M, Noguchi Y, Yoshitani K, Kawamata M, Masui K, et al. : Systematic review of motor evoked potentials monitoring during thoracic and thoracoabdominal aortic aneurysm open repair surgery: a diagnostic meta-analysis. J Anesth 30 : 1037-1050, 2016

73. Taniguchi M, Cedzich C, Schramm J : Modification of cortical stimulation for motor evoked potentials under general anesthesia: technical description. Neurosurgery 32 : 219-226, 1993

74. Taniguchi M, Nadstawek J, Pechstein U, Schramm J : Total intravenous anesthesia for improvement of intraoperative monitoring of somatosensory evoked potentials during aneurysm surgery. Neurosurgery 31 : 891- 
IOM for Pediatric Brain Tumor Surgery | Kim K, et al.

897; discussion 897, 1992

75. Thompson JE : Surgery for cerebrovascular insufficiency (stroke): with special emphasis on carotid endarterectomy. Springfield : Thomas, 1968

76. Wood CC, Spencer DD, Allison T, McCarthy G, Williamson PD, Goff WR : Localization of human sensorimotor cortex during surgery by cortical surface recording of somatosensory evoked potentials. J Neurosurg 68 :
99-111, 1988

77. Wylie EJ, Ehrenfeld WK : Extracranial occlusive cerebrovascular disease: diagnosis and management. Philadelphia : WB Saunders Company, 1970

78. Yanni DS, Ulkatan S, Deletis V, Barrenechea IJ, Sen C, Perin NI : Utility of neurophysiological monitoring using dorsal column mapping in intramedullary spinal cord surgery. J Neurosurg Spine 12 : 623-628, 2010 